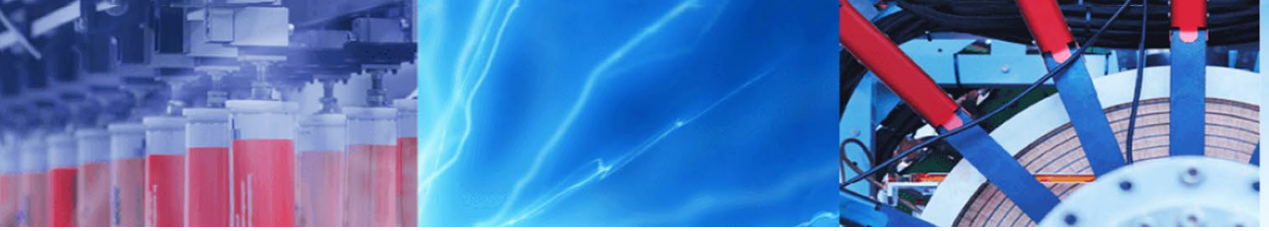

Research Article

\title{
Discontinuous Galerkin approximations in computational mechanics: hybridization, exact geometry and degree adaptivity
}

\author{
Matteo Giacomini ${ }^{1}$ (D) Ruben Sevilla ${ }^{2}$
}

(c) Springer Nature Switzerland AG 2019

\begin{abstract}
Discontinuous Galerkin (DG) discretizations with exact representation of the geometry and local polynomial degree adaptivity are revisited. Hybridization techniques are employed to reduce the computational cost of DG approximations and devise the hybridizable discontinuous Galerkin (HDG) method. Exact geometry described by non-uniform rational B-splines (NURBS) is integrated into HDG using the framework of the NURBS-enhanced finite element method. Moreover, optimal convergence and superconvergence properties of HDG-Voigt formulation in presence of symmetric second-order tensors are exploited to construct inexpensive error indicators and drive degree adaptive procedures. Applications involving the numerical simulation of problems in electrostatics, linear elasticity and incompressible viscous flows are presented. Moreover, this is done for both high-order HDG approximations and the lowest-order framework of face-centered finite volumes.
\end{abstract}

Keywords Hybridizable discontinuous Galerkin · Mixed formulation · Exact geometry · NURBS-enhanced finite element · Degree adaptivity · Superconvergence · Face-centered finite volume

\section{Introduction}

The importance of high-order approximations for the simulation of physical phenomena has been demonstrated in several fields of science and engineering, including electromagnetics [1,2] and flow problems [3, 4]. DG methods have shown great potential for the development of efficient high-order discretizations, exploiting modern parallel computing architectures and adaptive strategies for non-uniform degree approximations [1, 5-8]. Nevertheless, the duplication of unknowns in classical DG methods and their resulting higher computational cost have limited their application mostly to academic problems and only few attempts to perform large-scale DG simulations are available in the literature, see [9-11].

To remedy this issue, static condensation of finite element approximations [12] and hybridization of mixed methods [13] have received special attention in recent years. Following the rationale in [14], these concepts are applied to DG methods by defining the unknowns in each element as solution of a boundary value problem with Dirichlet data, whereas the interelement communication is handled by means of appropriate transmission conditions. Such approach leads to a wide range of hybrid discretization techniques [15] in which the only globally-coupled degrees of freedom of the problem are located on the mesh faces. The computational benefit of hybridization in the context of DG approximations has been analyzed in [16] in terms of floating-point operations. Other thorough numerical comparisons are detailed in $[17,18]$.

Contributions on hybrid methods may be subdivided in two main groups, relying either on primal or mixed formulations. The former includes: (1) classical DG methods in which the number of coupled degrees of freedom is

\footnotetext{
$\triangle$ Matteo Giacomini, matteo.giacomini@upc.edu | 'Laboratori de Càlcul Numèric (LaCàN), ETS de Ingenieros de Caminos, Canales y Puertos, Universitat Politècnica de Catalunya, Barcelona, Spain. ${ }^{2}$ Zienkiewicz Centre for Computational Engineering, College of Engineering, Swansea University, Swansea, Wales, UK.
} 
reduced simply by means of hybridization [19-21]; (2) the reduced stabilization approach exploiting a primal unknown approximated using a polynomial function of degree $k+1$ and a trace variable of polynomial degree $k$ to furtherly ease the computational burden $[22,23] ;(3)$ the hybrid high-order ( $\mathrm{HHO}$ ) method which introduces a local reconstruction operator to mimick the behavior of the gradient of the primal solution and an appropriate stabilization term $[24,25]$. It is worth recalling that the $\mathrm{HHO}$ method belongs to the family of hybridizable DG approaches and can be recasted in this framework by an appropriate definition of the involved stabilization operator [26].

Stemming from the work on the local DG method [27, 28], the hybridizable DG method proposed by Cockburn and coworkers relies on a mixed hybrid formulation [15], based on polynomial approximations discontinuous element-by-element [29]. The latter group thus includes all HDG formulations featuring the introduction of a mixed variable [30-39]. The advantage of directly approximating flux/stress via the introduction of a mixed variable is of special interest in the context of engineering problems in which quantities of interest usually rely on such information. Thus, in the following sections, these specific hybrid methods based on mixed formulations will be considered and, with an abuse of notation, they will be denoted generically as HDG approaches.

Hybrid discretization techniques have been successfully applied to several problems of engineering interest. In the context of computational fluid dynamics, HDG mixed formulations of the incompressible Navier-Stokes equations have been presented in $[36,40]$ and [41] using equal order and different order of polynomial approximations for the primal, mixed and hybrid variables, respectively. $\mathrm{HHO}$ formulations have been discussed in $[42,43]$. On the one hand, special emphasis has been devoted to the construction of pointwise divergence-free approximations in incompressible flows $[44,45]$. Recent results proposing a relaxed $H$ (div)-conforming discretization of the velocity field are available in $[46,47]$. On the other hand, extension to turbulent flows using implicit large eddy simulations [48] and the Spalart-Allmaras model $[49,50]$ and treatment of complex rheologies like quasi-Newtonian fluids [51] and viscoplastic materials [52] are active topics of investigations. First results of the application of hybrid discretization techniques to compressible flow problems are available in $[53,54]$.

Concerning linear elasticity, the strong enforcement of the symmetry of the stress tensor in HDG has been studied by different authors. A formulation using different degrees of polynomial approximation for the primal and hybrid variables has been discussed in [55]. In [56], an appropriate enrichment of the local discrete space of approximation via the $M$-decomposition framework is proposed to ensure optimal convergence of the mixed variable and superconvergence of the postprocessed one. An easy-to-implement alternative is represented by the HDG-Voigt approach introduced in [57] and detailed in Sect. 4 of the present contribution. In the context of nonlinear elasticity, hybrid methods based on primal formulations have shown promising results, see [58-60] for $\mathrm{HHO}$ applications to hyperelastic, plastic and elastoplastic regimes. The exploitation of HDG mixed formulations to simulate these phenomena is currently an open problem, as described in [61-63]. Moreover, results on fluid-structure interaction problems and arbitrary Lagrangian Eulerian formulations have been investigated in [64] and [65], respectively.

Other fields actively studied using hybrid discretization methods include subsurface flows $[66,67]$ and wave propagation phenomena [68], spanning from elastodynamics [69-71] to coastal water simulations [72], from Maxwell's equations [73] to acoustics [74], optics [75] and plasmonics $[76,77]$.

Besides the application of hybrid discretization methods to different physical problems, several efforts have been devoted in recent years to the construction of efficient strategies to exploit the numerical advantages of the above mentioned approaches. On the one hand, the flexibility of DG methods has been exploited to perform mesh refinement based on octrees [78], driven by adjointbased [79] and fully-computable [80] a posteriori error estimators. On the other hand, the possibility of using nonuniform polynomial degree approximations has been explored in [81] and [82, 83].

It is worth recalling that the accuracy of the functional approximation is strictly related to the one of the geometrical description of the domain. In this context, HDG for domains with curved boundaries have been analyzed in [84-87] via the extension to a fictitious subdomain, whereas a classical isoparametric framework has been developed for HHO in [88]. In [82, 83], the NEFEM paradigm is coupled with HDG to treat exact geometries described by means of NURBS. The strict relationship between geometrical and functional approximation error and its importance in the context of degree-adaptive procedures is further detailed in Sect. 3 of the present contribution.

Recently, different approaches to problems featuring unfitted interfaces have been proposed using immersed HDG formulations $[89,90]$, the extended HDG framework (X-HDG) which mutuates ideas from X-FEM to treat cut cells [91-93] and the cut-HHO method which relies on a cell agglomeration procedure and exploits the capability of $\mathrm{HHO}$ to handle generic mesh elements [94]. Moreover, numerical strategies to couple continuous Galerkin and HDG discretizations have also been recently proposed for mono- and multiphysics problems $[95,96]$. 
Concerning specific solution strategies for hybrid discretization methods, a parallel solver based on the iterative Schwarz method has been developed in [97], fast multigrid solvers have been employed in [98-100] and iterative approaches inspired by the Gauss-Seidel method have been discussed in [101, 102]. Moreover, tailored preconditioners for the hybrid DG method have been proposed in $[103,104]$ in the context of the Stokes equations.

This contribution presents an overview of some recent advances on HDG methods with application to different problems in computational mechanics, namely electrostatics, linear elasticity and incompressible viscous flow simulations. The rationale to devise an HDG mixed approximation of a second-order partial differential equation (PDE) is recalled in Sect. 2. In Sect. 3, the importance of accounting for the exact geometry described by means of NURBS is illustrated via the framework of NEFEM. An HDG-NEFEM discretization with degree adaptivity is thus discussed for an electrostatics problem. In Sect. 4, an application of HDG to linear elasticity is considered. Special attention is devoted to the construction of a formulation using a pointwise symmetric mixed variable, namely the strain rate tensor, via Voigt notation [105]. The resulting HDG-Voigt formulation is robust for nearly-incompressible materials and provides optimally-convergent stresses and superconvergent displacements which are exploited to construct local error indicators to perform degree adaptive procedures. Eventually, a lowest-order HDG approximation, the recently proposed FCFV method [106, 107], is devised to efficiently solve large-scale problems involving incompressible flows (Sect. 5). The FCFV method provides an LBB-stable discretization which is insensitive to mesh distortion and stretching and features first-order accurate fluxes without the need to perform a reconstruction procedure.

\section{The HDG rationale}

To recall the rationale of the HDG method, the Laplace equation is considered in an open bounded domain $\Omega \subset \mathbb{R}^{\mathrm{n}_{\mathrm{sd}}}, \mathrm{n}_{\mathrm{sd}}$ being the number of spatial dimensions,

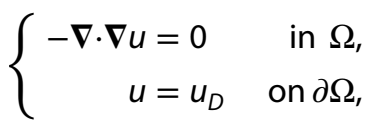

where $u$ and $u_{D}$ are the unknown variable and its imposed value on the boundary, respectively. From the point of view of modeling, Eq. (1) represents an electrostatic problem where $u$ is the unknown electric potential.

The standard HDG mixed formulation described in [108] is detailed. Recall that the main features of this HDG method is the introduction of a mixed variable, namely $\boldsymbol{q}=-\boldsymbol{\nabla} u$ allowing to rewrite a second-order PDE as a system of first-order PDEs, and of a hybrid variable û representing the trace of the primal unknown on the faces of the internal skeleton

$\Gamma:=\left[\bigcup_{e=1}^{\mathrm{n}_{\mathrm{e}} \mathrm{1}} \partial \Omega_{e}\right] \backslash \partial \Omega$,

where $\mathrm{n}_{\mathrm{el}}$ is the number of non-overlapping elements $\Omega_{e}, e=1, \ldots, \mathrm{n}_{\mathrm{e} 1}$ in which the domain is partitioned. Thus, Eq. (1) is rewritten as a system of first-order PDEs element-by-element

$$
\begin{cases}\boldsymbol{q}_{e}+\nabla u_{e}=\mathbf{0} & \text { in } \Omega_{e^{\prime}} e=1, \ldots, \mathrm{n}_{\mathrm{e} 1^{\prime}} \\ \boldsymbol{\nabla} \cdot \boldsymbol{q}_{e}=0 & \text { in } \Omega_{e^{\prime}} e=1, \ldots, \mathrm{n}_{\mathrm{e} 1^{\prime}} \\ u_{e}=u_{D} & \text { on } \partial \Omega_{e} \cap \partial \Omega, \\ u_{e}=\hat{u} & \text { on } \partial \Omega_{e} \backslash \partial \Omega\end{cases}
$$

with the following transmission conditions enforcing the continuity of the solution and of the fluxes across the interface $\Gamma$

$\left\{\begin{aligned} \llbracket u n \rrbracket & =\mathbf{0} & & \text { on } \Gamma, \\ \llbracket \widehat{\boldsymbol{n} \cdot \boldsymbol{q}} \rrbracket & =0 & & \text { on } \Gamma,\end{aligned}\right.$

where $\llbracket \odot \rrbracket=\odot_{i}+\odot_{,}$is the jump operator proposed in [109] as the sum of the values in the elements $\Omega_{i}$ and $\Omega_{l}$ on the right and on the left of the interface respectively, whereas the trace of the numerical flux is defined as

$\widehat{\boldsymbol{n} \cdot \boldsymbol{q}}:= \begin{cases}\boldsymbol{n} \cdot \boldsymbol{q}_{e}+\tau\left(u_{e}-u_{D}\right) & \text { on } \partial \Omega_{e} \cap \partial \Omega, \\ \boldsymbol{n} \cdot \boldsymbol{q}_{e}+\tau\left(u_{e}-\hat{u}\right) & \text { elsewhere, }\end{cases}$

with $\tau$ being an appropriate stabilization parameter [31-34, 36]. Note that the first transmission condition is automatically fulfilled owing to the Dirichlet boundary condition $u_{e}=\hat{u}$ imposed in the local problems on $\partial \Omega_{e} \backslash \partial \Omega$ and to the uniqueness of the hybrid variable $\hat{u}$ on each mesh face in $\partial \Omega_{e} \subset \Gamma$.

Thus, the HDG local problems are defined as follows: for $e=1, \ldots, \mathrm{n}_{\mathrm{el}}$ compute $\left(u_{e^{\prime}} \boldsymbol{q}_{e}\right) \in \mathcal{H}^{1}\left(\Omega_{e}\right) \times\left[\mathcal{H}\left(\operatorname{div} ; \Omega_{e}\right) ; \mathbb{R}^{\mathrm{n}_{\mathrm{sd}}}\right]$ such that

$\left\{\begin{array}{c}-\int_{\Omega_{e}} \boldsymbol{w} \cdot \boldsymbol{q}_{e} d \Omega+\int_{\Omega_{e}} \nabla \cdot w u_{e} d \Omega=\int_{\partial \Omega_{e} \cap \partial \Omega} \boldsymbol{n} \cdot \boldsymbol{w} u_{D} d \Gamma+\int_{\partial \Omega_{e} \mid \partial \Omega \Omega} \boldsymbol{n} \cdot \boldsymbol{w} \hat{u} d \Gamma, \\ \int_{\Omega_{e}} v \boldsymbol{\nabla} \cdot \boldsymbol{q}_{e} d \Omega+\int_{\partial \Omega_{e}} \tau v u_{e} d \Gamma=\int_{\partial \Omega_{e} \cap \partial \Omega} \tau v u_{D} d \Gamma+\int_{\partial \Omega_{e} \mid \partial \Omega} \tau v \hat{u} d \Gamma\end{array}\right.$

for all $(v, \boldsymbol{w}) \in \mathcal{H}^{1}\left(\Omega_{e}\right) \times\left[\mathcal{H}\left(\operatorname{div} ; \Omega_{e}\right) ; \mathbb{R}^{\mathrm{n}_{\mathrm{sd}}}\right], \quad$ where $\left[\mathcal{H}\left(\operatorname{div} ; \Omega_{e}\right) ; \mathbb{R}^{n_{s d}}\right]$ is the space of square integrable vectors of dimension $\mathrm{n}_{\mathrm{sd}}$ with square integrable divergence on $\Omega_{e}$. 
Following the notation in [108], the discrete functional spaces

$\mathcal{V}^{h}(\Omega):=\left\{v \in \mathcal{L}_{2}(\Omega):\left.v\right|_{\Omega_{e}} \in \mathcal{P}^{k}\left(\Omega_{e}\right) \forall \Omega_{e^{\prime}} e=1, \ldots, \mathrm{n}_{\mathrm{e}}\right\}$,

$\hat{\mathcal{V}}^{h}(S):=\left\{\hat{v} \in \mathcal{L}_{2}(S):\left.\hat{v}\right|_{\Gamma_{i}} \in \mathcal{P}^{k}\left(\Gamma_{i}\right) \forall \Gamma_{i} \subset S \subseteq \Gamma \cup \partial \Omega\right\}$,

are introduced for the HDG approximation. In Eq. (3), $\mathcal{P}^{k}\left(\Omega_{e}\right)$ (respectively, $\mathcal{P}^{k}\left(\Gamma_{i}\right)$ ) represents the space of polynomial functions of complete degree at most $k \geq 1$ in $\Omega_{e}$ (respectively, on $\Gamma_{i}$ ). Thus, for $e=1, \ldots, \mathrm{n}_{\mathrm{eI}}$ the HDG discrete local problem is: given $\hat{u}^{h}$ on $\Gamma$, find $\left(u_{e}^{h}, q_{e}^{h}\right) \in \mathcal{V}^{h}\left(\Omega_{e}\right) \times\left[\mathcal{V}^{h}\left(\Omega_{e}\right)\right]^{n_{s d}}$, approximating the pair $\left(u_{e}, \boldsymbol{q}_{e}\right)$, such that Eq. (2) holds for all $(v, \boldsymbol{w}) \in \mathcal{V}^{h}\left(\Omega_{e}\right) \times\left[\mathcal{V}^{h}\left(\Omega_{e}\right)\right]^{\mathrm{n}_{\mathrm{sd}}}$.

Remark 1 For each element $\Omega_{e^{\prime}} e=1, \ldots, \mathrm{n}_{\mathrm{e} 1}$, the primal, $u_{e^{\prime}}^{h}$ and mixed, $\boldsymbol{q}_{e^{\prime}}^{h}$ variables are determined as functions of the unknown hybrid variable $\hat{u}^{h}$ on $\partial \Omega_{e} \backslash \partial \Omega$. From the point of view of modeling, the HDG local problem establishes a relationship between the electric potential and electric field inside each element and the electric potential on the corresponding element boundary.

The HDG global problem is defined from the previously introduced transmission conditions: find $\hat{u} \in \mathcal{H}^{1 / 2}(\Gamma)$ such that

$$
\begin{aligned}
& \sum_{e=1}^{\mathrm{n}_{\mathrm{e} 1}}\left\{\int_{\partial \Omega_{e} \backslash \partial \Omega} \hat{v} \boldsymbol{n} \cdot \boldsymbol{q}_{e} d \Gamma\right. \\
& \left.\quad+\int_{\partial \Omega_{e} \backslash \partial \Omega} \tau \hat{v} u_{e} d \Gamma-\int_{\partial \Omega_{e} \backslash \partial \Omega} \tau \hat{v} \hat{u} d \Gamma\right\}=0,
\end{aligned}
$$

for all $\hat{v} \in \mathcal{L}_{2}(\Gamma)$, where $u_{e}$ and $\boldsymbol{q}_{e}$ are obtained from the local problems defined in Eq. (2).

The HDG discrete global problem is thus obtained solving the previous equation in the hybrid space introduced in (3), that is, find $\hat{u}^{h} \in \hat{\mathcal{V}}(\Gamma)$ such that Eq. (4) holds for all $\hat{v} \in \hat{\mathcal{V}}(\Gamma)$.

Recall that using equal order $k$ for the approximation of the primal, mixed and hybrid variables, HDG provides optimal convergence of order $k+1$ for all the unknowns [31]. Inspired by the work of Stenberg [110], this property is exploited to devise an inexpensive local postprocessing procedure leading to a superconvergent approximation of the primal variable $[35,82,108]$ : for $e=1, \ldots, \mathrm{n}_{\mathrm{e} 1}$, compute $u_{e}^{\star}$ using a polynomial approximation of degree $k+1$ such that

$$
\left\{\begin{array}{lr}
\boldsymbol{\nabla} \cdot \boldsymbol{\nabla} u_{e}^{\star}=-\boldsymbol{\nabla} \cdot \boldsymbol{q}_{e}^{h} & \text { in } \Omega_{e^{\prime}} \\
\boldsymbol{n} \cdot \boldsymbol{\nabla} u_{e}^{\star}=-\boldsymbol{n} \cdot \boldsymbol{q}_{e}^{h} & \text { on } \partial \Omega_{e^{\prime}}
\end{array}\right.
$$

with the solvability constraint

$\int_{\Omega_{e}} u_{e}^{\star} d \Omega=\int_{\Omega_{e}} u_{e}^{h} d \Omega$.

The computed $u_{e}^{\star}$ thus superconverges with order $k+2$ [111] and has been exploited to define a simple and inexpensive error indicator to perform degree adaptive procedures $[82,112,113]$.

Henceforth, the superscript ${ }^{h}$ identifying the discrete HDG solution will be omitted to ease readability and notation, if no risk of ambiguity is possible.

\section{HDG-NEFEM: exact geometry and degree adaptivity}

The possibility to easily implement a variable degree of approximation in DG methods has motivated the recent interest in degree adaptive processes for convectiondominated flow and wave propagation phenomena. In this context, the superconvergent property of HDG is especially attractive, as it allows to devise an inexpensive error indicator for a computed approximation $[82,112,113]$.

One aspect that has been traditionally ignored when proposing new degree adaptive procedures is the representation of the geometry. In an isoparametric formulation, a degree adaptive process requires communicating with the CAD model and regenerating the mesh, at least near the boundary, at each iteration. Nonetheless, the associated computational cost makes this strategy unfeasible for practical applications. Thus, it is common practice to represent the geometry with quadratic or cubic polynomials and change only the degree of the functional approximation during the adaptivity process, leading to subparametric and superparametric formulations [112, 113]. An alternative procedure based on the NEFEM rationale [114] is discussed here. The boundary of the computational domain is represented using the true CAD model, irrespective of the functional approximation used. The effort required to implement this approach is similar to the one employing subparametric or superparametric formulations: no communication with the CAD model or regeneration of the mesh are required, while the geometric uncertainty introduced by a polynomial description of the boundary of the domain is completely removed.

According to the framework described in Sect. 2, for a given distribution of the degree $k$ of the functional approximation, the global problem (4) is solved first to obtain the trace of the electric potential on the mesh edges/faces. Second, an element-by-element problem is solved to compute the value of the electric potential $u$ and its gradient, i.e. the electric field, in the elements, according to Eq. (2). Finally, an 
element-by-element postprocess is performed to obtain a superconvergent solution $u_{e}^{\star}$ by solving (5)-(6).

Following $[82,113]$, a measure of the error in each element $\Omega_{e}, e=1, \ldots, \mathrm{n}_{\mathrm{e} 1}$ is defined

$E_{e}^{u}=\left[\frac{1}{\left|\Omega_{e}\right|} \int_{\Omega_{e}}\left(u_{e}^{\star}-u_{e}\right)^{2} d \Omega\right]^{1 / 2}$.

Moreover, the local a priori error estimate derived in [115] for elliptic problems, states that the error in an element is bounded as

$\varepsilon_{e}=\left\|u-u_{h}\right\|_{\Omega_{e}} \leq C h_{e}^{k_{e}+1+n_{\mathrm{sd}} / 2}$.

By means of Richardson extrapolation, it is possible to estimate the unknown constant $C$ in Eq. (8), assuming that two values of the error, obtained with different degrees of approximation, are considered. In order to determine the change of degree required to achieve a desired error $\varepsilon$, first, an estimate of the error is devised using Eq. (7). Then, the target approximation degree is computed according to Eq. (8) by imposing a desired elemental error $\varepsilon_{e}$. As detailed in [82], the change of degree in the element $\Omega_{e}$ is thus given by

$\Delta k_{e}=\left\lceil\frac{\log \left(\varepsilon / E_{e}^{u}\right)}{\log \left(h_{e}\right)}\right\rceil, \quad e=1, \ldots, \mathrm{n}_{\mathrm{e} 1}$

where $\lceil\cdot\rceil$ is the ceiling function and $h_{e}$ is the non-dimensional characteristic size of the element $\Omega_{e}$.

The proposed degree adaptive process is tested by computing the electric field in a rectangular domain with a square inclusion, $\Omega=[-75,75] \times[-100,100] \backslash[-50,50]^{2}$.
A unit potential is imposed on the outer boundary and a zero potential on the inclusion. As it is common in practical engineering applications, the corners of the inclusion are rounded to eliminate the singularity induced by the re-entrant corners [116]. Specifically, a fillet defined using a small radius $r$ is introduced to increase the regularity of the boundary. Figure 1 shows the intensity of the electric field for three different geometries, with a fillet of radius $r=5, r=2$ and $r=1$ respectively.

The results clearly illustrate the change in the maximum intensity of the electric field (Fig. 1-top), as well as the localized variations at the corners in terms of the radius of the fillet (Fig. 1-bottom).

For the application of interest, a fillet of radius $r=1$ is considered. In this case, a fine mesh is thus required by isoparametric elements to capture the localized high curvature of the boundary around the corners and the degree adaptive process has to be coupled with mesh adaptation, leading to an $h p$-refinement strategy. With the proposed HDG-NEFEM approach, a coarse mesh of uniform element size is employed while preserving the exact representation of rounded corners. The degree adaptive process thus determines the required nonuniform degree of approximation to compute the solution with a desired tolerance $\varepsilon=0.5 \times 10^{-3}$, provided by the user a priori and represented by the target dashed line in Fig. 3. The resulting intensity of the electric field computed on a quarter of the domain and the distribution of the polynomial degree of approximation are depicted in Fig. 2.

The evolution of the estimated and exact errors for the proposed HDG-NEFEM approach is shown in Fig. 3 (left) and compared against the estimated and exact errors for an isoparametric approach. It is important to note that the usual
Fig. 1 Top: intensity of the electric field for three different radii of the fillet. From left to right: $r=5, r=2$ and $r=1$. Bottom: detail of the top-right corner of the inclusion and the computed electric field
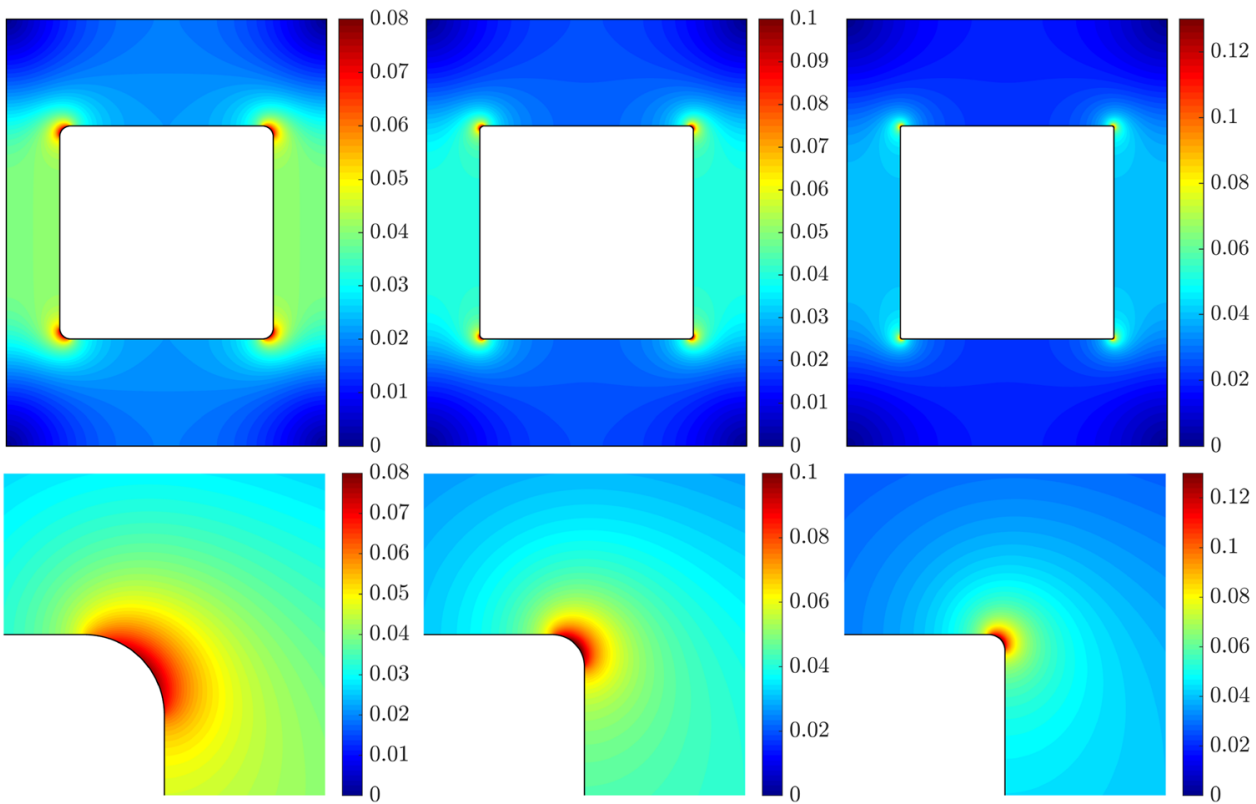

SN Applied Sciences A SPRINGer NATURE journal 
isoparametric strategy presents two major drawbacks. First, each iteration involving a modification of the polynomial degree in the elements with the rounded corner requires communication with the CAD model and regeneration of the distributions of nodes for the curved elements. Second, the change in geometry induced by the change in the degree of the functional approximation is not able to decrease the error towards the imposed tolerance. The nonsmooth representation of the geometry, i.e. only $\mathcal{C}^{0}$ between the elements, entails that the numerical approximation of $u$ presents a nonphysical singularity and the degree adaptive process does not provide an optimal solution. In contrast, with the proposed HDG-NEFEM approach the error decreases monotonically until the desired tolerance is achieved.

To further illustrate the benefits of the proposed HDGNEFEM approach, a degree adaptive process is performed next with standard high-order elements avoiding the costly communication with the CAD model. To this end, the geometry is represented with cubic polynomials and during the degree adaptive process only the degree of the functional approximation of the solution is changed.

Figure 3 (right) shows the evolution of the estimated and exact errors. The results clearly show that despite the adaptive process stops in eight iterations because the estimated error has reached the desired tolerance, the exact error is far

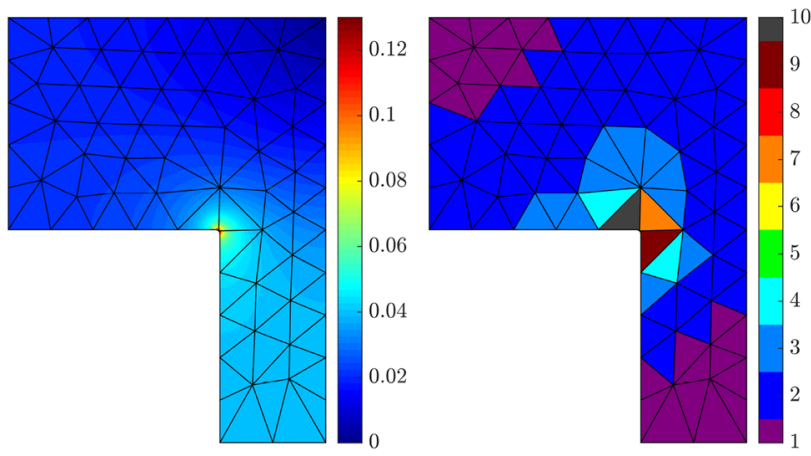

Fig. 2 Left: intensity of the electric field computed with the proposed HDG-NEFEM approach. Right: distribution of the approximation degree after eight iterations of the degree adaptive process from being close to the desired error. This indicates that the adaptive process is actually converging to the solution of a different problem where the geometry is represented with polynomials and remains unchanged during the adaptive iterations. It is worth noticing that using a fixed polynomial approximation of the geometry in a degree adaptive context has been extensively utilized in the literature, see [112, 113], but this simple example demonstrates the limitations of such approach.

\section{HDG-Voigt formulation in continuum mechanics and local error indicators}

In continuum mechanics, the strong enforcement of the symmetry of the stress tensor is associated with the pointwise fulfillment of the conservation of angular momentum. It is well-known that the classical HDG mixed formulation suffers from suboptimal convergence when low-order discretizations of symmetric second-order tensors are involved $[34,37]$. To remedy this issue, several techniques have been proposed in the context of hybrid discretization techniques $[25,41,55,56,117]$. The HDG-Voigt formulation introduced in $[57,118]$ exploits Voigt notation for second-order tensors, see [105], to strongly enforce symmetry by storing solely $\mathrm{m}_{\mathrm{sd}}:=\mathrm{n}_{\mathrm{sd}}\left(\mathrm{n}_{\mathrm{sd}}-1\right) / 2$ non-redundant off-diagonal components of the stress tensor in a vector form $\sigma_{v}$, namely,

$\sigma_{\mathrm{v}}:= \begin{cases}{\left[\sigma_{11}, \sigma_{22}, \sigma_{12}\right]^{T}} & \mathrm{n}_{\mathrm{sd}}=2, \\ {\left[\sigma_{11}, \sigma_{22}, \sigma_{33}, \sigma_{12}, \sigma_{13}, \sigma_{23}\right]^{T}} & \mathrm{n}_{\mathrm{sd}}=3,\end{cases}$

where $\mathrm{n}_{\mathrm{sd}}$ is the number of spatial dimensions of the problem.

Consider a domain $\Omega \subset \mathbb{R}^{\mathrm{n}_{\text {sd }}}$ such that $\partial \Omega=\Gamma_{D} \cup \Gamma_{N}$ and $\Gamma_{D} \cap \Gamma_{N}=\emptyset$ and the following system of equations describing the behavior of a continuum medium

$$
\begin{cases}-\nabla_{\mathrm{s}}^{T} \sigma_{\mathrm{v}}=\boldsymbol{s} & \text { in } \Omega, \\ \sigma_{\mathrm{v}}=\mathbf{D}(E, v) \boldsymbol{\nabla}_{\mathrm{s}} \boldsymbol{u} & \text { in } \Omega, \\ \boldsymbol{u}=\boldsymbol{u}_{D} & \text { on } \Gamma_{D^{\prime}} \\ \mathbf{N}^{T} \sigma_{\mathrm{v}}=\boldsymbol{g} & \text { on } \Gamma_{N^{\prime}}\end{cases}
$$

Fig. 3 Evolution of the exact and estimated errors as a function of the number of iterations in the degree adaptive procedure. Left: isoparametric and NEFEM elements. Right: high-order elements with a fixed approximation of the geometry using cubic polynomials
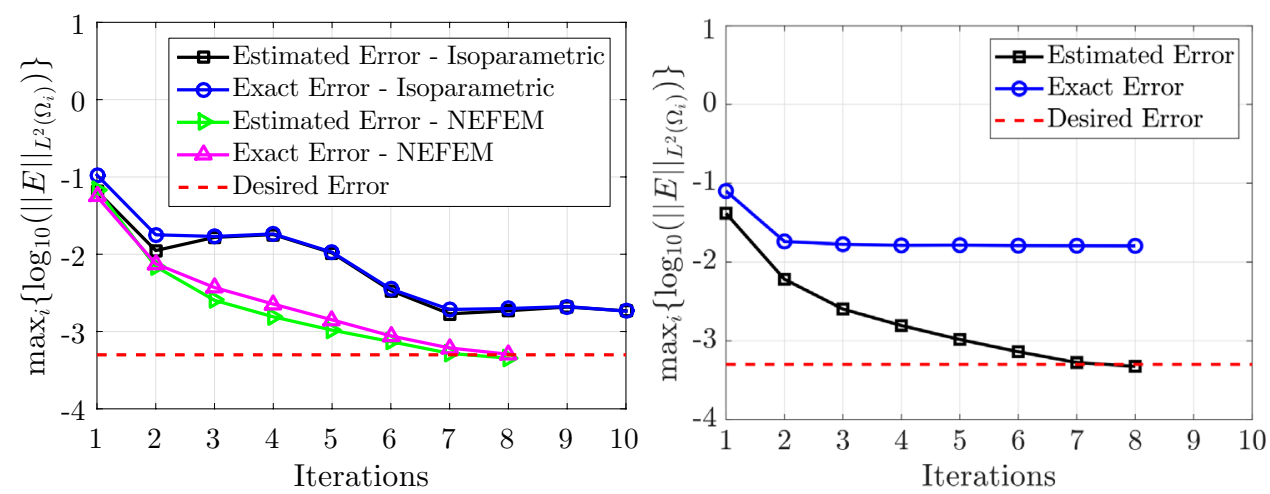
where $\boldsymbol{u}$ is the unknown displacement field, $\boldsymbol{s}$ is the external body force and $\boldsymbol{u}_{D}, \boldsymbol{g}$ are the imposed displacement and traction on the boundary, respectively. The $\mathrm{m}_{\mathrm{sd}} \times \mathrm{n}_{\mathrm{sd}}$ matrices $\boldsymbol{\nabla}_{\mathrm{s}}$ and $\mathbf{N}$ account for the linearized symmetric gradient operator and the normal direction to the boundary and have the following form
$\mathbf{N}^{T} \mathbf{D}^{1 / 2} \boldsymbol{L}_{e}:= \begin{cases}\mathbf{N}^{T} \mathbf{D}^{1 / 2} \boldsymbol{L}_{e}+\tau\left(\boldsymbol{u}_{e}-\boldsymbol{u}_{D}\right) & \text { on } \partial \Omega_{e} \cap \Gamma_{D^{\prime}} \\ \mathbf{N}^{T} \mathbf{D}^{1 / 2} \boldsymbol{L}_{e}+\tau\left(\boldsymbol{u}_{e}-\widehat{\boldsymbol{u}}\right) & \text { elsewhere. }\end{cases}$

Note that owing to the Voigt framework, the mixed variable utilized in the HDG formulation is the pointwise

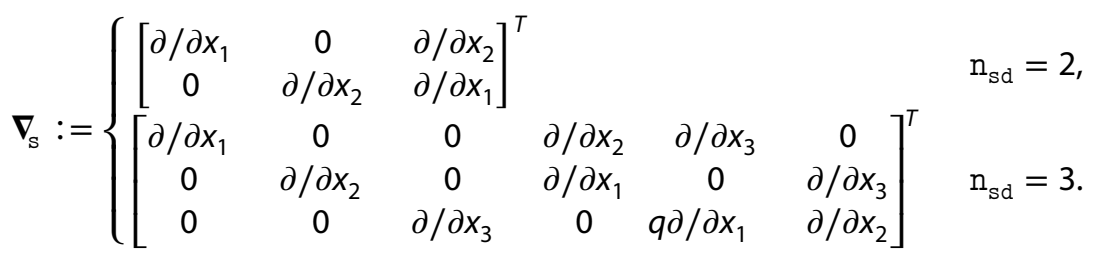

$\mathbf{N}:=\left\{\begin{array}{ccc}{\left[\begin{array}{ccccc}n_{1} & 0 & n_{2} \\ 0 & n_{2} & n_{1}\end{array}\right]^{T}} & & \\ {\left[\begin{array}{cccccc}n_{1} & 0 & 0 & n_{2} & n_{3} & 0 \\ 0 & n_{2} & 0 & n_{1} & 0 & n_{3} \\ 0 & 0 & n_{3} & 0 & n_{1} & n_{2}\end{array}\right]^{T} \quad \mathrm{n}_{\mathrm{sd}}=3 .}\end{array}\right.$

The relationship between the stress tensor and the displacement field is expressed by means of the Hooke's law for linear elastic homogeneous materials, $\sigma_{\mathrm{v}}=\mathbf{D}(E, v) \boldsymbol{\nabla}_{\mathrm{s}} \boldsymbol{u}$, where the matrix $\mathbf{D}$ describes the mechanical behavior of the solid as a function of Young's modulus $E$ and Poisson's ratio $v$, according to the classical definitions in [105].

Introducing a symmetric mixed variable $\boldsymbol{L}_{e}$ for the discretization of the strain rate tensor, the linear elasticity problem in Eq. (11) is split into a set of $n_{e 1}$ local problems that define the primal and mixed variables $\left(\boldsymbol{u}_{e}, \boldsymbol{L}_{e}\right)$ as functions of the hybrid variable $\hat{u}$ representing the trace of the displacement field on the edges/faces of the mesh, namely

$$
\begin{cases}\boldsymbol{L}_{e}+\mathbf{D}^{1 / 2} \nabla_{\mathrm{s}} \boldsymbol{u}_{e}=\mathbf{0} & \text { in } \Omega_{e^{\prime}} e=1, \ldots, \mathrm{n}_{\mathrm{e} 1} \\ \nabla_{\mathrm{s}}^{T} \mathbf{D}^{1 / 2} \boldsymbol{L}_{e}=\boldsymbol{s} & \text { in } \Omega_{e^{\prime}} e=1, \ldots, \mathrm{n}_{\mathrm{e} 1} \\ \boldsymbol{u}_{e}=\boldsymbol{u}_{D} & \text { on } \partial \Omega_{e} \cap \Gamma_{D^{\prime}} \\ \boldsymbol{u}_{e}=\widehat{u} & \text { on } \partial \Omega_{e} \backslash \Gamma_{D^{\prime}}\end{cases}
$$

and a global problem imposing the Neumann boundary condition and the transmission conditions to enforce inter-element continuity of the solution and the tractions

$$
\begin{cases}\mathbf{N}^{T} \mathbf{D}^{1 / 2} \boldsymbol{L}_{e}=-\boldsymbol{g} & \text { on } \Gamma, \\ \llbracket \boldsymbol{u} \otimes \boldsymbol{n} \rrbracket=\mathbf{0} & \text { on } \Gamma, \\ \llbracket \mathbf{N}^{T \mathbf{D}^{1 / 2}} \boldsymbol{L}_{e} \rrbracket=\mathbf{0} & \text { on } \Gamma,\end{cases}
$$

where $\boldsymbol{n}$ is the outward normal vector to the faces of the internal skeleton $\Gamma$ and $\mathbf{N}^{\top} \mathbf{D}^{1 / 2} L_{e}$ is the trace of the numerical flux, defined as a function of $\hat{\boldsymbol{u}}$ and the stabilization parameter $\tau$ symmetric strain rate tensor, the conservation of angular momentum is fulfilled pointwise in each mesh element and physical tractions are imposed on the Neumann boundary. Following the HDG rationale, first, the global problem in Eq. (14) is solved to obtain $\hat{\boldsymbol{u}}$ on the internal skeleton $\Gamma$ and on the Neumann boundary $\Gamma_{N}$. Then, the primal and mixed variables $\left(\boldsymbol{u}_{e}, \boldsymbol{L}_{e}\right)$ are computed element-by-element by solving the HDG local problems in Eq. (13) independently in each $\Omega_{e}, e=1, \ldots, \mathrm{n}_{\mathrm{el}}$. Eventually, the following postprocessing procedure is devised: for $e=1, \ldots, \mathrm{n}_{\mathrm{eI}}$ compute a displacement field $\boldsymbol{u}_{e}^{\star}$ using a polynomial approximation of degree $k+1$ such that

$\left\{\begin{aligned} & \boldsymbol{\nabla}_{\mathrm{s}}^{T} \mathbf{D}^{1 / 2} \boldsymbol{\nabla}_{\mathrm{s}} \boldsymbol{u}_{e}^{\star}=-\boldsymbol{\nabla}_{\mathrm{s}}^{T} \boldsymbol{L}_{e} \quad \text { in } \boldsymbol{\Omega}_{e^{\prime}} \\ & \mathbf{N}^{T} \mathbf{D}^{1 / 2} \boldsymbol{\nabla}_{\mathrm{s}} \boldsymbol{u}_{e}^{\star}=-\mathbf{N}^{T} \boldsymbol{L}_{e} \text { on } \partial \Omega_{e^{\prime}}\end{aligned}\right.$

with the solvability constraint in Eq. (6) to remove the underdetermination due to rigid body translations and

$\int_{\Omega_{e}} \boldsymbol{\nabla} \times \boldsymbol{u}_{e}^{\star} d \Omega=\int_{\partial \Omega_{e}} \boldsymbol{u}_{e} \cdot \boldsymbol{t} d \Gamma$,

to account for rigid body rotations, where $\boldsymbol{t}$ is the tangential direction to the boundary $\partial \Omega_{e}$.

It is worth recalling that HHO and HDG, that is, both primal and mixed formulations of hybrid discretization methods display a robust behavior for nearly incompressible materials and do not experience locking phenomena $[25,37,38]$. The discussed HDG-Voigt strategy inherits such property. Nonetheless, classical HDG methods using approximations with equal-order polynomials of degree $k$ for all the variables experience suboptimal behavior for $k<3$. On the contrary, the proposed HDGVoigt formulation provides a discretization with optimal convergence of order $k+1$ for $\boldsymbol{u}, \boldsymbol{L}$ and $\hat{\boldsymbol{u}}$, even in case of low-order polynomial approximations. Thus, in this context, the advantages of using the HDG-Voigt formulation are twofold. On the one hand, an approximation of the strain rate tensor is directly obtained from the 
mixed formulation without the need to postprocess the primal variable of the problem. On the other hand, the resulting method provides optimally convergent stress and superconvergent displacement field using a nodalbased approximation for all the variables [57] and without resorting to different interpolation degrees [55] or to the enrichment of the local discrete spaces discussed in [56].

The HDG-Voigt formulation is tested on a well-known benchmark test for bending-dominated elastic problems, the Cook's membrane [119]. The domain consists of a tapered plate clamped on the left end and subject to a vertical shear load $\boldsymbol{g}=(0,1 / 16)$ on the opposite end, whereas zero tractions are imposed on the top and bottom parts of the boundary. Following the problem setup in [120], a nearly incompressible material with Young's modulus $E=1.12499998125$ and Poisson's ratio $v=0.499999975$ is considered. Figure 4 shows the displacement of the mid-point of the right end of the membrane for linear, quadratic and cubic elements on both quadrilateral and triangular meshes.

The results display the convergence to the reference value, taken from [120], even for low-order triangular elements, showing the robustness of the HDG-Voigt formulation in the incompressible limit.

Exploiting the optimal convergence of order $k+1$ of the discretized strain rate tensor $L_{e}$ and the postprocessing procedure discussed in $[57,118]$ to resolve the underdetermination due to rigid body motions, a superconvergent approximation $\boldsymbol{u}_{e}^{\star}$ of the displacement field is constructed. Thus, the error indicator in Eq. (7) is computed starting from the approximated primal and postprocessed displacement fields. Alternatively, a local error indicator based on the strain rate tensor

$E_{e}^{L}=\left[\frac{1}{\left|\Omega_{e}\right|} \int_{\Omega_{e}}\left(\nabla_{\mathrm{s}} u_{e}^{\star}-\nabla_{\mathrm{s}} u_{e}\right) \cdot\left(\nabla_{\mathrm{s}} \boldsymbol{u}_{e}^{\star}-\nabla_{\mathrm{s}} \boldsymbol{u}_{e}\right) d \Omega\right]^{1 / 2}$

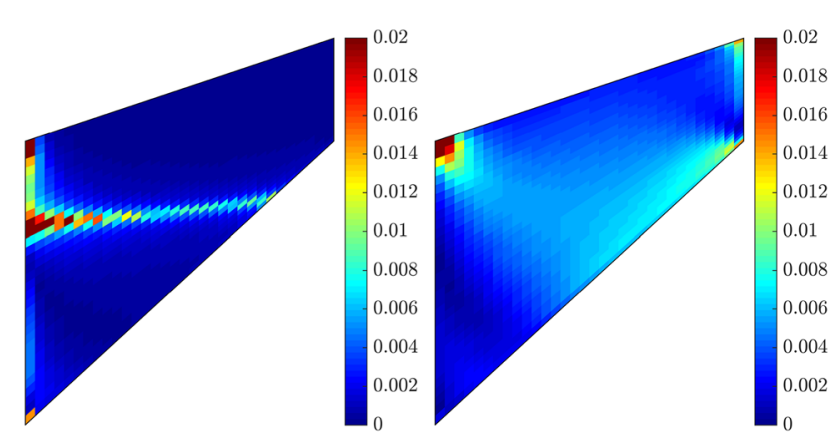

Fig. 5 Error indicator based on the displacement field (left) and the strain rate tensor (right)

can be used when a certain level of accuracy is required on the stress tensor rather than on the displacement field [83].

Figure 5 shows a comparison of the error indicators (7) and (18) for the displacement field and the strain rate tensor, respectively. The different information captured by each error indicator is clearly observed. In particular, it is straightforward to observe that the error indicator based on the strain rate tensor is able to provide information about regions where a concentration of stress is present. This information is of great interest in engineering applications, e.g. for the optimal design of elastic structures [121].

\section{FCFV: lowest-order HDG method for large-scale problems}

One of the major challenges that current techniques in computational mechanics face when confronted to industrial applications is proving their ability to efficiently solve large-scale problems in a reliable and robust way. Despite the numerous advantages in terms of accuracy, efficient treatment of convection-dominated phenomena in flow problems and flexibility for parallelization, the adoption
Fig. 4 Convergence of the displacement of the mid-point of the right end of Cook's membrane as a function of the number of degrees of freedom of the HDG discretization, using polynomial approximations of degree $k=1,2,3$. Left: quadrilateral elements. Right: triangular elements
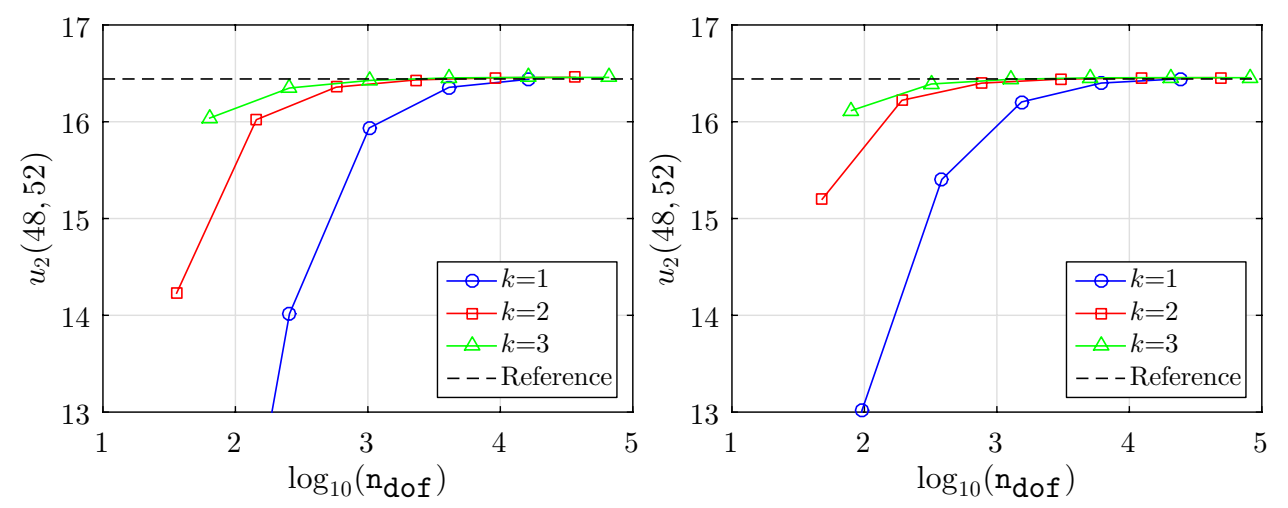
of high-order methods by the industry is still limited, partially due to the difficulty to generate high-order curvilinear meshes of complex configurations [122]. Starting from the framework discussed above, a novel efficient and robust finite volume (FV) rationale has been proposed in $[106,107]$.

In order to describe this approach, an incompressible Stokes flow is considered

$$
\begin{cases}-\boldsymbol{\nabla} \cdot\left(\nu \boldsymbol{\nabla} \boldsymbol{u}-p \mathbf{I}_{\mathrm{n}_{\mathrm{sd}}}\right)=\boldsymbol{s} & \text { in } \Omega, \\ \boldsymbol{\nabla} \cdot \boldsymbol{u}=0 & \text { in } \Omega, \\ \boldsymbol{u}=\boldsymbol{u}_{D} & \text { on } \Gamma_{D^{\prime}} \\ \left(\nu \boldsymbol{\nabla} \boldsymbol{u}-p \mathbf{I}_{\mathrm{n}_{\mathrm{sd}}}\right) \boldsymbol{n}=\boldsymbol{g} & \text { on } \Gamma_{N^{\prime}}\end{cases}
$$

where the pair $(\boldsymbol{u}, p)$ represents the unknown velocity and pressure fields, $v>0$ is the viscosity of the fluid, $\mathbf{I}_{\mathrm{n}_{\mathrm{sd}}}$ is the $\mathrm{n}_{\mathrm{sd}} \times \mathrm{n}_{\mathrm{sd}}$ identity matrix and $\boldsymbol{s}, \boldsymbol{u}_{D}, \boldsymbol{g}$ respectively are the source term, the imposed velocity and pseudo-tractions, see [123], on the boundary.

Following the HDG rationale introduced in Sect. 2, the FCFV local and global problems for the Stokes equations are introduced. More precisely, in each cell $\Omega_{e^{\prime}} e=1, \ldots, \mathrm{n}_{\mathrm{e} 1}$, it holds

$$
\begin{cases}\boldsymbol{L}_{e}+\sqrt{v} \boldsymbol{\nabla} \boldsymbol{u}_{e}=\mathbf{0} & \text { in } \Omega_{e^{\prime}} e=1, \ldots, \mathrm{n}_{\mathrm{e} 1} \\ \boldsymbol{\nabla} \cdot\left(\sqrt{v} \boldsymbol{L}_{e}+p_{e} \mathbf{I}_{\mathrm{n}_{\mathrm{sd}}}\right)=\boldsymbol{s} & \text { in } \Omega_{e^{\prime}} e=1, \ldots, \mathrm{n}_{\mathrm{e} 1} \\ \boldsymbol{\nabla} \cdot \boldsymbol{u}_{e}=0 & \text { in } \Omega_{e^{\prime}} e=1, \ldots, \mathrm{n}_{\mathrm{e} 1} \\ \boldsymbol{u}_{e}=\boldsymbol{u}_{D} & \text { on } \partial \Omega_{e} \cap \Gamma_{D^{\prime}} \\ \boldsymbol{u}_{e}=\hat{\boldsymbol{u}} & \text { on } \partial \Omega_{e} \backslash \Gamma_{D^{\prime}}\end{cases}
$$

with the following additional constraint to remove the underdetermination of pressure due to the Dirichlet boundary conditions imposed in Eq. (20)

$\int_{\partial \Omega_{e}} p_{e} d \Gamma=\left|\partial \Omega_{e}\right| \rho_{e}$.

The FCFV global problem features the Neumann boundary conditions and the transmission conditions enforcing inter-element continuity of the solution and the fluxes, as previously detailed for the HDG method

$$
\begin{cases}\left(\sqrt{v} \boldsymbol{L}_{e}+p_{e} \mathbf{I}_{\mathrm{n}_{\mathrm{sd}}}\right) \boldsymbol{n}=-\boldsymbol{g} & \text { on } \Gamma, \\ \llbracket \boldsymbol{u} \otimes \boldsymbol{n} \rrbracket=\mathbf{0} & \text { on } \Gamma, \\ \llbracket\left(\sqrt{v} \boldsymbol{L}_{e}+p_{e} \mathbf{I}_{\mathrm{n} \mathrm{sd}}\right) \boldsymbol{n} \rrbracket=\mathbf{0} & \text { on } \Gamma,\end{cases}
$$

where the numerical normal flux on the boundary is defined as $\left(\sqrt{v} \boldsymbol{L}_{e}+p_{e} \mathbf{I}_{\mathrm{n}_{\mathrm{sd}}}\right) \boldsymbol{n}:= \begin{cases}\left(\sqrt{v} \boldsymbol{L}_{e}+p_{e} \mathbf{I}_{\mathrm{n}_{\mathrm{s}}}\right) \boldsymbol{n}+\tau\left(\boldsymbol{u}_{e}-\boldsymbol{u}_{D}\right) & \text { on } \partial \Omega_{e} \cap \Gamma_{D}, \\ \left.\sqrt{v} \boldsymbol{L}_{e}+p_{e} \mathbf{I}_{\mathrm{n}_{\mathrm{sa}}}\right) \boldsymbol{n}+\tau\left(\boldsymbol{u}_{e}-\widehat{\boldsymbol{u}}\right) & \text { elsewhere. }\end{cases}$

Moreover, the incompressibility constraint is expressed in weak form as

$\int_{\partial \Omega_{e} \backslash \Gamma_{D}} \hat{\boldsymbol{u}} \cdot \boldsymbol{n} d \Gamma+\int_{\partial \Omega_{e} \cap \Gamma_{D}} \boldsymbol{u}_{D} \cdot \boldsymbol{n} d \Gamma=0 \quad$ for $e=1, \ldots, \mathrm{n}_{\mathrm{e} 1}$.

FCFV may be interpreted as the lowest-order HDG mixed method which employs a constant degree of approximation in each cell for the velocity $\boldsymbol{u}_{e}$, the pressure $p_{e}$ and the mixed variable $\boldsymbol{L}_{e}$, representing the gradient of velocity, a constant degree of approximation on each edge/face for the velocity $\hat{u}$ and a constant value $\rho_{e}$ for the mean pressure in each cell. Moreover, the FCFV global and local problems are discretized using a quadrature with one integration point located in the centroid of the cell or face and in the midpoint of the edge.

FCFV solves the problem in two phases [106, 107]. First, by applying the divergence theorem to Eq. (20) and exploiting the definition of the numerical normal flux on the boundary in Eq. (23), a set of $n_{\mathrm{el}}$ local integral problems is obtained

$$
\begin{aligned}
-\int_{\Omega_{e}} \boldsymbol{L}_{e} d \Omega & =\int_{\partial \Omega_{e} \cap \Gamma_{D}} \sqrt{v} \boldsymbol{u}_{D} \otimes \boldsymbol{n} d \Gamma+\int_{\partial \Omega_{e} \backslash \Gamma_{D}} \sqrt{v} \hat{\boldsymbol{u}} \otimes \boldsymbol{n} d \Gamma, \\
\int_{\partial \Omega_{e}} \tau \boldsymbol{u}_{e} d \Gamma & =\int_{\Omega_{e}} \boldsymbol{s} d \Omega+\int_{\partial \Omega_{e} \cap \Gamma_{D}} \tau \boldsymbol{u}_{D} d \Gamma+\int_{\partial \Omega_{e} \backslash \Gamma_{D}} \tau \hat{\boldsymbol{u}} d \Gamma, \\
\int_{\partial \Omega_{e}} p_{e} d \Gamma & =\left|\partial \Omega_{e}\right| \rho_{e} .
\end{aligned}
$$

Note that the divergence theorem applied to the incompressibility constraint in Eq. (20) leads to Eq. (24), which is thus omitted from the local problem since only the global unknown $\hat{\boldsymbol{u}}$ is involved. Moreover, the last equation of the previous system directly stems from Eq. (21).

It is worth noticing that the equations of the FCFV local problem decouple and a closed-form expression of all the variables as functions of the velocity $\hat{\boldsymbol{u}}$ on the boundary $\partial \Omega_{e} \backslash \Gamma_{D}$ and the mean value $\rho_{e}$ of the pressure inside the element $\Omega_{e}$ is obtained. The previously determined elemental expressions of $\left(\boldsymbol{u}_{e^{\prime}} p_{e^{\prime}} \boldsymbol{L}_{e}\right)$ are employed to solve the FCFV global problem (22) with the incompressibility constraint in Eq. (24), namely

$$
\begin{aligned}
& \sum_{e=1}^{\mathrm{n}_{e}}\left\{\int_{\partial \Omega_{e} \mid \Gamma_{D}} \sqrt{v} \boldsymbol{L}_{e} \boldsymbol{n} d \Gamma+\int_{\partial \Omega_{e} \mid \Gamma_{D}} p_{e} \boldsymbol{n} d \Gamma+\int_{\partial \Omega_{e} \backslash \Gamma_{D}} \tau \boldsymbol{u}_{e} d \Gamma\right. \\
& \left.-\int_{\partial \Omega_{e} \backslash \Gamma_{D}} \tau \hat{\boldsymbol{u}} d \Gamma\right\}=-\sum_{e=1}^{\mathrm{n}_{\mathrm{e} 1}} \int_{\partial \Omega_{e} \cap \Gamma_{N}} \boldsymbol{g} d \Gamma, \\
& \quad \int_{\partial \Omega_{e} \mid \Gamma_{D}} \hat{\boldsymbol{u}} \cdot \boldsymbol{n} d \Gamma=-\int_{\partial \Omega_{e} \cap \Gamma_{D}} \boldsymbol{u}_{D} \cdot \boldsymbol{n} d \Gamma=0 \quad \text { for } e=1, \ldots, \mathrm{n}_{\mathrm{e} 1} .
\end{aligned}
$$


Fig. 6 Pressure field on 39 particles modelling RBCs immersed in an incompressible Stokes flow in a channel and streamlines of the velocity field

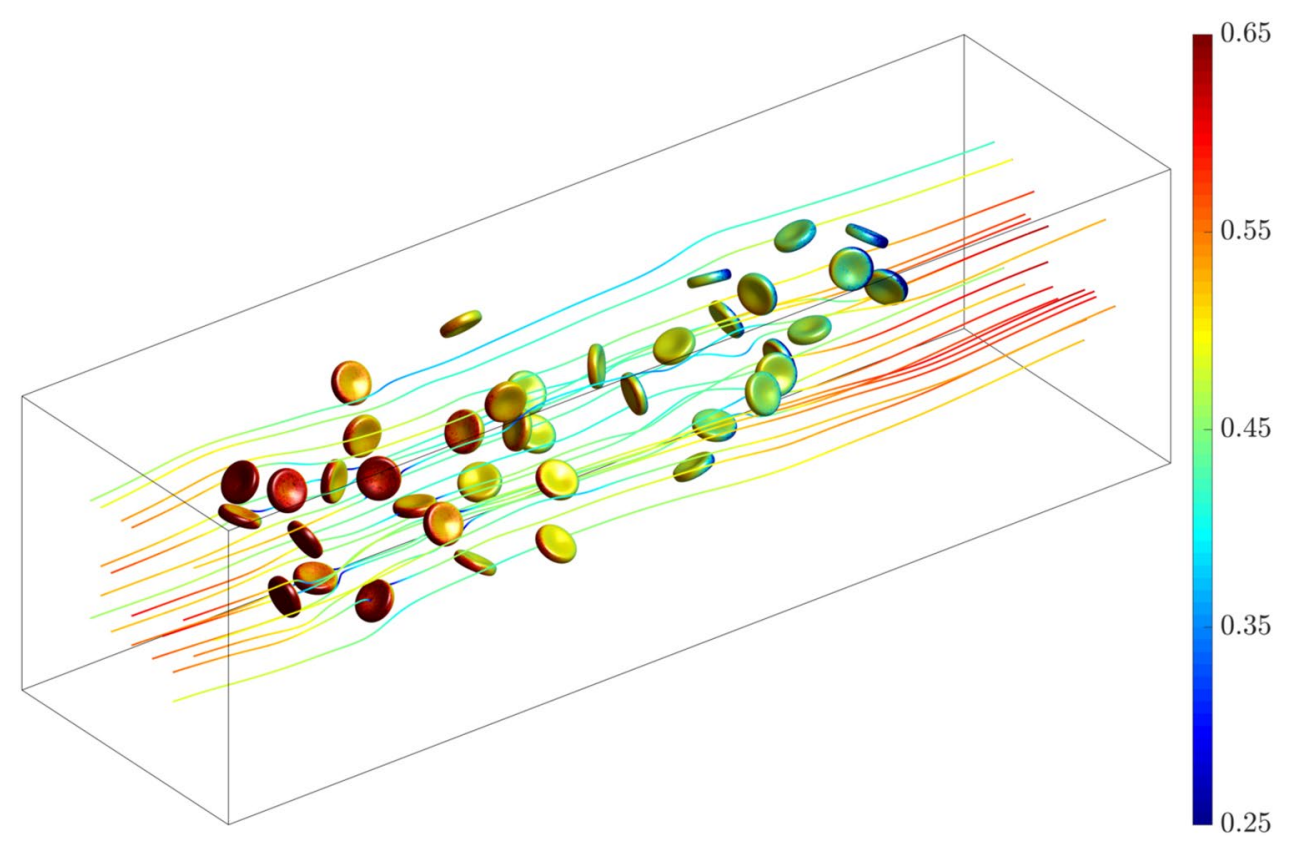

The resulting linear system obtained from the FCFV discretization of Eq. (26) is symmetric and features a saddlepoint structure with $n_{f c} n_{s d}+n_{e l}$ unknowns, being $n_{f c}$ the number of internal and Neumann edges/faces. The FCFV global problem has a sparse block structure allowing a computationally efficient implementation, see [106]. Moreover, FCFV local computations to determine velocity, pressure and gradient of velocity in the centroid of each cell solely involve elementary operations cell-by-cell for which modern parallel architectures can be exploited.

FCFV inherits the approximation properties of the corresponding high-order HDG formulation from which it is derived. More precisely, optimal first-order convergence is obtained for velocity, pressure and gradient of velocity. In addition, contrary to other mixed finite element methods, with the FCFV it is possible to use the same space of approximation for both velocity and pressure, circumventing the so-called Ladyzhenskaya-Babuška-Brezzi (LBB) condition.

Compared to other FV methods, the FCFV provides firstorder accuracy of the solution and its gradient without the need to perform flux reconstruction as in the context of cell-centered and vertex-centered finite volumes [124, 125]. Furthermore, the accuracy of the FCFV method is preserved in presence of unstructured meshes, with distorted and stretched cells $[106,107]$. This is of major importance when solving problems in complex geometries as other FV methods lose accuracy and optimal convergence properties when non-orthogonal and anisotropic cells are introduced in the computational mesh [124, 125].

To highlight the efficiency of the proposed FCFV method, a Stokes flow is simulated in a channel with 39 rigid particles in the shape of red-blood cells (RBCs). A parabolic velocity profile modelling an undisturbed flow is imposed on the inlet and on the outlet of the channel, whereas a no-slip boundary condition is imposed on the remaining walls and on the surface of the particles.

The computational domain $\Omega=[-10,25] \times[-5,5]$ $\times[-5,5] \backslash \mathcal{B}$, where $\mathcal{B}$ is the union of the $39 \mathrm{RBC}$, is discretized using an unstructured mesh of $8,972,888$ tetrahedral cells, 35,891,552 nodes and 17,523,981 internal faces. The FCFV global system for the mesh configuration under analysis features $61,544,832$ unknowns. The simulation was performed using a code developed in Matlab ${ }^{\circledR}$. The computation of all the elemental contributions to the global system took $51 \mathrm{~min}$ whereas $18 \mathrm{~min}$ were required for the assembly of the matrix. The solution of the linear system was performed using the Matlab ${ }^{\circledR}$ biconjugate gradient method in a single processor and without preconditioner. Eventually, the evaluation of the element-by-element solution in all 61 millions elements took 7 min using a single processor.

The pressure distribution on the surface of the RBCs and the velocity streamlines are presented in Fig. 6 .

Figure 7 displays the magnitude of the velocity field at three different sections of the computational domain.

\section{Concluding remarks}

Three recent contributions to HDG are discussed in this paper. First, the HDG-NEFEM paradigm exploits the description of the boundary of the domain via NURBS to construct an HDG approximation with exact geometry 
Fig. 7 Magnitude of the velocity field of an incompressible Stokes flow in a channel with 39 particles modelling RBCs.

Section plane for $y=-3$ (top), $y=0$ (center) and $y=3$ (bottom)

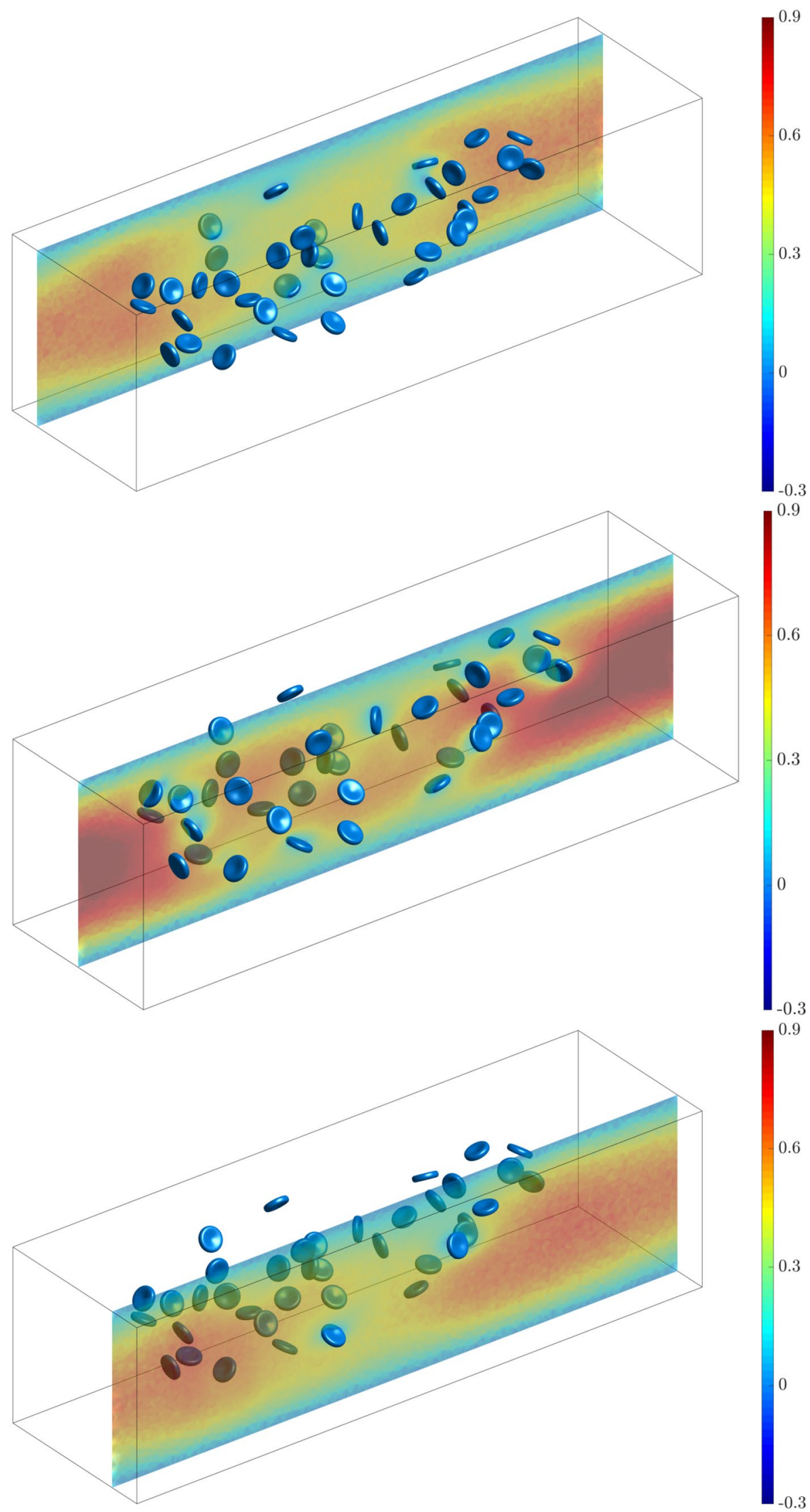


and to devise an efficient and robust degree adaptivity strategy. Second, the HDG-Voigt formulation is utilized in the context of continuum mechanics to devise an HDG method with pointwise symmetric mixed variable, namely the strain rate tensor. The resulting formulation allows to achieve optimal convergence and superconvergence properties even for low-order polynomial approximations and, consequently, to compute local error indicators based on either the displacement or the stress field. Third, the FCFV rationale proposes a fast implementation of the lowest-order HDG method. The resulting finite volume paradigm is reconstruction-free, robust to mesh distortion and element stretching and is able to efficiently tackle large-scale problems. Ongoing investigations focus on the application of the discussed strategies to nonlinear problems of interest in engineering applications and to the simulation of transient phenomena.

Acknowledgements This work is partially supported by the European Union's Horizon 2020 research and innovation programme under the Marie Skłodowska-Curie actions (Grant No. 675919 and 764636) and the Spanish Ministry of Economy and Competitiveness (Grant No. DPI2017-85139-C2-2-R). The first author also gratefully acknowledges the financial support provided by Generalitat de Catalunya (Grant No. 2017-SGR-1278).

\section{Compliance with ethical standards}

Conflict of interest The authors declare that they have no conflict of interest.

\section{References}

1. Hesthaven JS, Warburton T (2002) Nodal high-order methods on unstructured grids: I. Time-domain solution of Maxwell's equations. J Comput Phys 181(1):186-221

2. Dawson M, Sevilla R, Morgan K (2018) The application of a high-order discontinuous Galerkin time-domain method for the computation of electromagnetic resonant modes. Appl Math Model 55:94-108

3. Bassi F, Rebay S (1997) High-order accurate discontinuous finite element solution of the 2D Euler equations. J Comput Phys 138(2):251-285

4. Abgrall R, Ricchiuto M (2017) High-order methods for CFD. In: Stein E, Borst R, Hughes TJ (eds) Encyclopedia of computational mechanics, 2nd edn. https://doi.org/10.1002/9781119176817. ecm 2112

5. Cockburn B, Karniadakis GE, Shu C-W (2000) The development of discontinuous Galerkin methods. In: Discontinuous Galerkin methods (Newport, RI, 1999), volume 11 of lecture notes computer science engineering. Springer, Berlin, pp 3-50

6. Rivière B (2008) Discontinuous Galerkin methods for solving elliptic and parabolic equations. Society for Industrial and Applied Mathematics, Philadelphia

7. Di Pietro DA, Ern A (2012) Mathematical aspects of discontinuous Galerkin methods, vol 69. Springer, Heidelberg
8. Cangiani A, Dong Z, Georgoulis EH, Houston P (2017) hp-Version discontinuous Galerkin methods on polygonal and polyhedral meshes. Springer, Berlin

9. Crivellini A, Bassi F (2011) An implicit matrix-free discontinuous Galerkin solver for viscous and turbulent aerodynamic simulations. Comput Fluids 50(1):81-93

10. Frank HM, Munz C-D (2016) Direct aeroacoustic simulation of acoustic feedback phenomena on a side-view mirror. J Sound Vib 371:132-149

11. Fehn N, Wall WA, Kronbichler M (2019) A matrix-free highorder discontinuous Galerkin compressible Navier-Stokes solver: a performance comparison of compressible and incompressible formulations for turbulent incompressible flows. Int J Numer Methods Fluids 89(3):71-102

12. Guyan RJ (1965) Reduction of stiffness and mass matrices. AIAA J 3(2):380-380

13. Fraeijs de Veubeke B (1965) Displacement and equilibrium models in the finite element method. In: Zienkiewicz OC, Holister GS (eds) Stress analysis. Wiley, New York, pp 145-197

14. Cockburn B (2016) Static condensation, hybridization, and the devising of the HDG methods. In: Barrenechea GR, Brezzi F, Cangiani A, Georgoulis EH (eds) Building bridges: connections and challenges in modern approaches to numerical partial differential equations. Springer, Cham, pp 129-177

15. Brezzi F, Fortin M (1991) Mixed and hybrid finite elements methods. Springer series in computational mathematics. Springer, Berlin

16. Huerta A, Angeloski A, Roca X, Peraire J (2013) Efficiency of high-order elements for continuous and discontinuous Galerkin methods. Int J Numer Methods Eng 96(9):529-560

17. Kirby R, Sherwin SJ, Cockburn B (2011) To CG or to HDG: a comparative study. J Sci Comput 51(1):183-212

18. Woopen M, Balan A, May G, Schütz J (2014) A comparison of hybridized and standard DG methods for target-based hpadaptive simulation of compressible flow. Comput Fluids 98:3-16

19. Egger H, Schöberl J (2009) A hybrid mixed discontinuous Galerkin finite-element method for convection-diffusion problems. IMA J Numer Anal 30(4):1206-1234

20. Egger $\mathrm{H}$, Waluga $\mathrm{C}$ (2012) A hybrid mortar method for incompressible flow. Int J Numer Anal Model 9(4):793-812

21. Egger $\mathrm{H}$, Waluga $\mathrm{C}$ (2012) hp analysis of a hybrid DG method for Stokes flow. IMA J Numer Anal 33(2):687-721

22. Oikawa I (2015) A hybridized discontinuous Galerkin method with reduced stabilization. J Sci Comput 65(1):327-340

23. Oikawa I (2016) Analysis of a reduced-order HDG method for the Stokes equations. J Sci Comput 67(2):475-492

24. Di Pietro DA, Ern A, Lemaire S (2014) An arbitrary-order and compact-stencil discretization of diffusion on general meshes based on local reconstruction operators. Comput Methods Appl Math 14(4):461-472

25. Di Pietro DA, Ern A (2015) A hybrid high-order locking-free method for linear elasticity on general meshes. Comput Methods Appl Mech Eng 283:1-21

26. Cockburn B, Di Pietro DA, Ern A (2016) Bridging the hybrid high-order and hybridizable discontinuous Galerkin methods. ESAIM: M2AN 50(3):635-650

27. Cockburn B, Shu C-W (1998) The local discontinuous Galerkin method for time-dependent convection-diffusion systems. SIAM J Numer Anal 35(6):2440-2463

28. Cockburn B, Dong B, Guzmán J (2008) A superconvergent LDG-hybridizable Galerkin method for second-order elliptic problems. Math Comput 77(264):1887-1916

29. Cockburn B, Gopalakrishnan J (2004) A characterization of hybridized mixed methods for second order elliptic problems. SIAM J Numer Anal 42(1):283-301 
30. Cockburn B, Gopalakrishnan J (2009) The derivation of hybridizable discontinuous Galerkin methods for Stokes flow. SIAM J Numer Anal 47(2):1092-1125

31. Cockburn B, Gopalakrishnan J, Lazarov R (2009) Unified hybridization of discontinuous Galerkin, mixed, and continuous Galerkin methods for second order elliptic problems. SIAM J Numer Anal 47(2):1319-1365

32. Nguyen NC, Peraire J, Cockburn B (2009) An implicit highorder hybridizable discontinuous Galerkin method for linear convection-diffusion equations. J Comput Phys 228(9):3232-3254

33. Nguyen NC, Peraire J, Cockburn B (2009) An implicit highorder hybridizable discontinuous Galerkin method for nonlinear convection-diffusion equations. J Comput Phys 228(23):8841-8855

34. Cockburn B, Nguyen NC, Peraire J (2010) A comparison of HDG methods for Stokes flow. J Sci Comput 45(1-3):215-237

35. Nguyen NC, Peraire J, Cockburn B (2010) A hybridizable discontinuous Galerkin method for Stokes flow. Comput Methods Appl Mech Eng 199(9-12):582-597

36. Nguyen NC, Peraire J, Cockburn B (2011) An implicit highorder hybridizable discontinuous Galerkin method for the incompressible Navier-Stokes equations. J Comput Phys 230(4):1147-1170

37. Soon S-C, Cockburn B, Stolarski HK (2009) A hybridizable discontinuous Galerkin method for linear elasticity. Int J Numer Methods Eng 80(8):1058-1092

38. Fu G, Cockburn B, Stolarski H (2015) Analysis of an HDG method for linear elasticity. Int J Numer Methods Eng 102(3-4):551-575

39. Schütz J, May G (2013) A hybrid mixed method for the compressible Navier-Stokes equations. J Comput Phys 240:58-75

40. Cesmelioglu A, Cockburn B, Qiu W (2017) Analysis of a hybridizable discontinuous Galerkin method for the steady-state incompressible Navier-Stokes equations. Math Comput 86(306):1643-1670

41. Qiu W, Shi K (2016) A superconvergent HDG method for the incompressible Navier-Stokes equations on general polyhedral meshes. IMA J Numer Anal 36(4):1943-1967

42. Di Pietro DA, Krell S (2018) A hybrid high-order method for the steady incompressible Navier-Stokes problem. J Sci Comput 74(3):1677-1705

43. Botti L, Di Pietro DA, Droniou J (2019) A hybrid high-order method for the incompressible Navier-Stokes equations based on Temam's device. J Comput Phys 376:786-816

44. Lehrenfeld C, Schöberl J (2016) High order exactly divergencefree hybrid discontinuous Galerkin methods for unsteady incompressible flows. Comput Methods Appl Mech Eng 307:339-361

45. Rhebergen S, Wells GN (2018) A hybridizable discontinuous Galerkin method for the Navier-Stokes equations with pointwise divergence-free velocity field. J Sci Comput 76(3):1484-1501

46. Lederer P, Lehrenfeld C, Schöberl J (2018) Hybrid discontinuous Galerkin methods with relaxed $\mathrm{H}$ (div)-conformity for incompressible flows. Part I. SIAM J Numer Anal 56(4):2070-2094

47. Lederer PL, Lehrenfeld C, Schöberl J (2019) Hybrid discontinuous Galerkin methods with relaxed $\mathrm{H}$ (div)-conformity for incompressible flows. Part II. ESAIM: M2AN 53(2):503-522

48. Fernandez P, Nguyen NC, Peraire J (2017) The hybridized discontinuous Galerkin method for implicit large-eddy simulation of transitional turbulent flows. J Comput Phys 336:308-329

49. Moro D, Nguyen NC, Peraire J (2011) Navier-Stokes solution using hybridizable discontinuous Galerkin methods. In: 20th AIAA computational fluid dynamics conference. AIAA

50. Peters EL, Evans JA (2019) A divergence-conforming hybridized discontinuous Galerkin method for the incompressible
Reynolds averaged Navier-Stokes equations. Int J Numer Methods Fluids 91:112-133. https://doi.org/10.1002/fld.4745

51. Gatica GN, Sequeira FA (2015) Analysis of an augmented HDG method for a class of quasi-Newtonian Stokes flows. J Sci Comput 65(3):1270-1308

52. Cascavita KL, Bleyer J, Chateau X, Ern A (2018) Hybrid discretization methods with adaptive yield surface detection for Bingham pipe flows. J Sci Comput 77(3):1424-1443

53. Peraire J, Nguyen NC, Cockburn B (2010) A hybridizable discontinuous Galerkin method for the compressible Euler and Navier-Stokes equations. AIAA Paper 363:2010

54. Williams DM (2018) An entropy stable, hybridizable discontinuous Galerkin method for the compressible Navier-Stokes equations. Math Comput 87(309):95-121

55. Qiu W, Shen J, Shi K (2018) An HDG method for linear elasticity with strong symmetric stresses. Math Comput 87(309):69-93

56. Cockburn B, Fu G (2017) Devising superconvergent HDG methods with symmetric approximate stresses for linear elasticity by M-decompositions. IMA J Numer Anal 38(2):566-604

57. Sevilla R, Giacomini M, Karkoulias A, Huerta A (2018) A superconvergent hybridisable discontinuous Galerkin method for linear elasticity. Int J Numer Methods Eng 116(2):91-116

58. Abbas M, Ern A, Pignet N (2018) Hybrid high-order methods for finite deformations of hyperelastic materials. Comput Mech 62(4):909-928

59. Abbas M, Ern A, Pignet N (2019) A hybrid high-order method for incremental associative plasticity with small deformations. Comput Methods Appl Mech Eng 346:891-912

60. Abbas M, Ern A, Pignet N (2019) A hybrid high-order method for finite elastoplastic deformations within a logarithmic strain framework. Int J Numer Methods Eng. https://doi.org/10.1002/ nme.6137

61. Kabaria H, Lew AJ, Cockburn B (2015) A hybridizable discontinuous galerkin formulation for non-linear elasticity. Comput Methods Appl Mech Eng 283:303-329

62. Cockburn B, Shen J (2019) An algorithm for stabilizing hybridizable discontinuous Galerkin methods for nonlinear elasticity. Results Appl Math 1:100001

63. Terrana S, Nguyen NC, Bonet J, Peraire J (2019) A hybridizable discontinuous Galerkin method for both thin and 3D nonlinear elastic structures. Comput Methods Appl Mech Eng 352:561-585

64. Sheldon JP, Miller ST, Pitt JS (2016) A hybridizable discontinuous Galerkin method for modeling fluid-structure interaction. J Comput Phys 326:91-114

65. Fidkowski KJ (2016) A hybridized discontinuous Galerkin method on mapped deforming domains. Comput Fluids 139:80-91

66. Fabien MS, Knepley MG, Rivière BM (2018) A hybridizable discontinuous Galerkin method for two-phase flow in heterogeneous porous media. Int J Numer Methods Eng 116(3):161-177

67. Costa-Solé A, Ruiz-Gironés E, Sarrate J (2019) An HDG formulation for incompressible and immiscible two-phase porous media flow problems. Int J Comput Fluid Dyn 33(4):137-148

68. Fernandez P, Christophe A, Terrana S, Nguyen NC, Peraire J (2018) Hybridized discontinuous Galerkin methods for wave propagation. J Sci Comput 77(3):1566-1604

69. Bonnasse-Gahot M, Calandra H, Diaz J, Lanteri S (2017) Hybridizable discontinuous Galerkin method for the 2-D frequencydomain elastic wave equations. Geophys J Int 213(1):637-659

70. Terrana S, Vilotte JP, Guillot L (2017) A spectral hybridizable discontinuous Galerkin method for elastic-acoustic wave propagation. Geophys J Int 213(1):574-602

71. Hungria A, Prada D, Sayas F-J (2017) HDG methods for elastodynamics. Comput Math Appl 74(11):2671-2690 
72. Samii A, Dawson C (2018) An explicit hybridized discontinuous Galerkin method for Serre-Green-Naghdi wave model. Comput Methods Appl Mech Eng 330:447-470

73. Christophe A, Descombes S, Lanteri S (2018) An implicit hybridized discontinuous Galerkin method for the 3D time-domain Maxwell equations. Appl Math Comput 319:395-408

74. Schoeder S, Kronbichler M, Wall WA (2018) Arbitrary high-order explicit hybridizable discontinuous Galerkin methods for the acoustic wave equation. J Sci Comput 76(2):969-1006

75. Li L, Lanteri S, Mortensen NA, Wubs M (2017) A hybridizable discontinuous Galerkin method for solving nonlocal optical response models. Comput Phys Commun 219:99-107

76. Vidal-Codina F, Nguyen NC, Oh S-H, Peraire J (2018) A hybridizable discontinuous Galerkin method for computing nonlocal electromagnetic effects in three-dimensional metallic nanostructures. J Comput Phys 355:548-565

77. Vidal-Codina F, Nguyen NC, Peraire J (2018) Computing parametrized solutions for plasmonic nanogap structures. J Comput Phys 366:89-106

78. Samii A, Michoski C, Dawson C (2016) A parallel and adaptive hybridized discontinuous Galerkin method for anisotropic nonhomogeneous diffusion. Comput Methods Appl Mech Eng 304:118-139

79. Woopen M, May G, Schütz J (2014) Adjoint-based error estimation and mesh adaptation for hybridized discontinuous Galerkin methods. Int J Numer Methods Fluids 76(11):811-834

80. Ainsworth M, Fu G (2018) Fully computable a posteriori error bounds for hybridizable discontinuous Galerkin finite element approximations. J Sci Comput 77(1):443-466

81. Hoermann JM, Bertoglio C, Kronbichler M, Pfaller MR, Chabiniok R, Wall WA (2018) An adaptive hybridizable discontinuous Galerkin approach for cardiac electrophysiology. Int J Numer Methods Biomed Eng 34(5):e2959

82. Sevilla R, Huerta A (2018) HDG-NEFEM with degree adaptivity for Stokes flows. J Sci Comput 77(3):1953-1980

83. Sevilla R (2019) HDG-NEFEM for two dimensional linear elasticity. Comput Struct 220:69-80

84. Cockburn B, Solano M (2012) Solving Dirichlet boundary-value problems on curved domains by extensions from subdomains. SIAM J Sci Comput 34(1):A497-A519

85. Cockburn B, Solano M (2014) Solving convection-diffusion problems on curved domains by extensions from subdomains. J Sci Comput 59(2):512-543

86. Solano M, Vargas F (2019) A high order HDG method for stokes flow in curved domains. J Sci Comput 79(3):1505-1533

87. Sánchez-Vizuet T, Solano ME (2019) A hybridizable discontinuous Galerkin solver for the Grad-Shafranov equation. Comput Phys Commun 235:120-132

88. Botti L, Di Pietro DA (2018) Assessment of hybrid high-order methods on curved meshes and comparison with discontinuous Galerkin methods. J Comput Phys 370:58-84

89. Dong H, Wang B, Xie Z, Wang L-L (2016) An unfitted hybridizable discontinuous Galerkin method for the Poisson interface problem and its error analysis. IMA J Numer Anal 37(1):444-476

90. Qiu W, Solano M, Vega P (2016) A high order HDG method for curved-interface problems via approximations from straight triangulations. J Sci Comput 69(3):1384-1407

91. Gürkan C, Sala-Lardies E, Kronbichler M, Fernández-Méndez S (2016) Extended hybridizable discontinous Galerkin (X-HDG) for void problems. J Sci Comput 66(3):1313-1333

92. Gürkan C, Kronbichler M, Fernández-Méndez S (2017) Extended hybridizable discontinuous Galerkin with Heaviside enrichment for heat bimaterial problems. J Sci Comput 72(2):542-567
93. Gürkan C, Kronbichler M, Fernández-Méndez S (2019) Extended hybridizable discontinuous Galerkin for incompressible flow problems with unfitted meshes and interfaces. Int J Numer Methods Eng 117(7):756-777

94. Burman E, Ern A (2018) An unfitted hybrid high-order method for elliptic interface problems. SIAM J Numer Anal 56(3):1525-1546

95. Paipuri M, Tiago C, Fernández-Méndez S (2019) Coupling of continuous and hybridizable discontinuous Galerkin methods: application to conjugate heat transfer problem. J Sci Comput 78(1):321-350

96. La Spina A, Giacomini M, Huerta A (2019) Hybrid coupling of CG and HDG discretizations based on Nitsche's method. https ://arxiv.org/abs/1906.10711 (Submitted)

97. Gander MJ, Hajian S (2018) Analysis of Schwarz methods for a hybridizable discontinuous Galerkin discretization: the manysubdomain case. Math Comput 87(312):1635-1657

98. Schütz J, Aizinger V (2017) A hierarchical scale separation approach for the hybridized discontinuous Galerkin method. J Comput Appl Math 317:500-509

99. Kronbichler M, Wall W (2018) A performance comparison of continuous and discontinuous Galerkin methods with fast multigrid solvers. SIAM J Sci Comput 40(5):A3423-A3448

100. Fabien M, Knepley M, Mills R, Rivière B (2019) Manycore parallel computing for a hybridizable discontinuous Galerkin nested multigrid method. SIAM J Sci Comput 41(2):C73-C96

101. Muralikrishnan S, Tran M, Bui-Thanh T (2017) iHDG: an iterative HDG framework for partial differential equations. SIAM J Sci Comput 39(5):S782-S808

102. Muralikrishnan S, Tran M, Bui-Thanh T (2018) An improved iterative HDG approach for partial differential equations. J Comput Phys 367:295-321

103. Rhebergen S, Wells GN (2018) Preconditioning of a hybridized discontinuous Galerkin finite element method for the Stokes equations. J Sci Comput 77(3):1936-1952

104. Barrenechea GR, Bosy M, Dolean V, Nataf F, Tournier P-H (2018) Hybrid discontinuous Galerkin discretisation and domain decomposition preconditioners for the Stokes problem. Comput Methods Appl Math. https://doi.org/10.1515/ cmam-2018-0005

105. Fish J, Belytschko T (2007) A first course in finite elements. Wiley, New York

106. Sevilla R, Giacomini M, Huerta A (2018) A face-centred finite volume method for second-order elliptic problems. Int J Numer Methods Eng 115(8):986-1014

107. Sevilla R, Giacomini M, Huerta A (2019) A locking-free facecentred finite volume (FCFV) method for linear elastostatics. Comput Struct 212:43-57

108. Sevilla R, Huerta A (2016) Tutorial on hybridizable discontinuous Galerkin (HDG) for second-order elliptic problems. In: Schröder J, Wriggers P (eds) Advanced finite element technologies, volume 566 of CISM International Centre for Mechanical Sciences. Springer, Berlin, pp 105-129

109. Montlaur A, Fernández-Méndez S, Huerta A (2008) Discontinuous Galerkin methods for the Stokes equations using divergence-free approximations. Int J Numer Methods Fluids 57(9):1071-1092

110. Stenberg R (1988) A family of mixed finite elements for the elasticity problem. Numer Math 53(5):513-538

111. Cockburn B, Dong B, Guzmán J (2008) A superconvergent LDGhybridizable Galerkin method for second-order elliptic problems. Math Comput 77(264):1887-1916

112. Giorgiani G, Fernández-Méndez S, Huerta A (2013) Hybridizable discontinuous Galerkin p-adaptivity for wave propagation problems. Int J Numer Methods Fluids 72(12):1244-1262 
113. Giorgiani G, Fernández-Méndez S, Huerta A (2014) Hybridizable discontinuous Galerkin with degree adaptivity for the incompressible Navier-Stokes equations. Comput Fluids 98:196-208

114. Sevilla R, Fernández-Méndez S, Huerta A (2008) NURBSenhanced finite element method (NEFEM). Int J Numer Methods Eng 76(1):56-83

115. Díez P, Huerta A (1999) A unified approach to remeshing strategies for finite element $h$-adaptivity. Comput Methods Appl Mech Eng 176(1-4):215-229

116. Krähenbühl L, Buret $F$, Perrussel $R$, Voyer $D$, Dular $P$, Péron $V$, Poignard C (2011) Numerical treatment of rounded and sharp corners in the modeling of 2D electrostatic fields. J Microw Optoelectron Electromagn Appl 10:66-81

117. Cockburn B, Fu G, Qiu W (2017) A note on the devising of superconvergent HDG methods for Stokes flow by M-decompositions. IMA J Numer Anal 37(2):730-749

118. Giacomini M, Karkoulias A, Sevilla R, Huerta A (2018) A superconvergent HDG method for Stokes flow with strongly enforced symmetry of the stress tensor. J Sci Comput 77(3):1679-1702

119. Cook RD, Malkus DS, Plesha ME, Witt RJ (2002) Concepts and applications of finite element analysis. Wiley, New York

120. Auricchio F, da Veiga LB, Lovadina C, Reali A (2005) An analysis of some mixed-enhanced finite element for plane linear elasticity. Comput Methods Appl Mech Eng 194(27-29):2947-2968

121. Allaire G, Dapogny C (2014) A linearized approach to worstcase design in parametric and geometric shape optimization. Math Models Methods Appl Sci 24(11):2199-2257

122. Xie ZQ, Sevilla R, Hassan O, Morgan K (2013) The generation of arbitrary order curved meshes for 3D finite element analysis. Comput Mech 51(3):361-374

123. Donea J, Huerta A (2003) Finite element methods for flow problems. Wiley, New York

124. Diskin B, Thomas JL, Nielsen EJ, Nishikawa H, White JA (2010) Comparison of node-centered and cell-centered unstructured finite-volume discretizations: viscous fluxes. AIAA J 48(7):1326

125. Diskin B, Thomas JL (2011) Comparison of node-centered and cell-centered unstructured finite-volume discretizations: inviscid fluxes. AIAA J 49(4):836-854

Publisher's Note Springer Nature remains neutral with regard to jurisdictional claims in published maps and institutional affiliations. 